\title{
Influential Article Review - Across Regional Innovation Systems and Commercial Communities, The Benefits of Variation Are Numerous
}

\author{
Lola Howell \\ Cora Ortiz
}

\begin{abstract}
Alessandra Kendall
This paper examines innovation systems. We present insights from a highly influential paper. Here are the highlights from this paper: Today, interesting and important interconnections have been made that promise great leaps forward for innovation systems and entrepreneurial ecosystems - especially operating at the regional or sub-national level of the space economy. Of course, there are politics in such relationships. Most notable are those that are critical of anything that "interferes" with market hegemony (neoliberal bias) which has weakened commitments such as those pioneered in South Korea in the early years of the twenty-first century, later to be followed by numerous Scandinavian policy experiments (Denmark, Norway and Sweden) as well as austerity policy to enlarge regional governance spaces, as in Germany and France (not to mention the dismantling in 2010 of Anglo-regional governance in the UK). Meanwhile, however, it can be seen that good progress in regional innovation policy and entrepreneurial accomplishment at regional level proceeds apace. This is known as the "co-operative bias" in contemporary political economy. Entrepreneurial ecosystems and regional innovation systems are excellent examples of "generative growth" mechanisms (floated in Cooke, P, Generative Growth, Knowledge Economies and Sustainable Development: implications for Regional Foresight Policy, 2002) as a counter to Romer-style individualistic endogenous growth theory. The paper explores the virtues of variety against those of linearity in innovation and entrepreneurial ecosystems in exemplary empirical instances. For our overseas readers, we then present the insights from this paper in Spanish, French, Portuguese, and German.
\end{abstract}

\section{SUMMARY}

- We have reached the three main discussion points of the foregoing analysis, as a prelude to the three concluding points to which they are related. The first of these opens up a more holistic, integrated and communal mode of institutional economic practice. These privileges - to an extent - the individualistic profit-seeking entrepreneur, though it prefers the more neutral and various characteristics of the enterprise as the vehicle for value analysis and realization. This correlates with the notion of "generative growth", a more evolutionary, communal and socially interactive learning process than the narrower theory of endogenous growth. This is reductionist as well as individualistic and determined by its main focus on the profit motive par excellence. These would construct their purposive actions in relation to other enterprises that facilitated the achievement of the higher purpose, such as the lightbulb, the smartphone or the prosthetic hip or knee joint. There

104 Journal of Strategic Innovation and Sustainability Vol. 16(4) 2021 
is relatively little of this "ancestry analysis" by comparison with the volumes of studies of "the world's oldest firm" in entrepreneurship studies. There may be some risk attached to formulating "industry interface" or "cross-over" inter- sector or inter-cluster studies but from an innovation standpoint much innovation has this "related variety" character including "revealed" value occurring by accident as much as design. However, it is the purposiveness that justifies the risk for search and selection potential.

- And as a final discussion point, in regard to our discussion of statics and dynamics in relation to enterprise ecosystems. Second, one of the insights of evolutionary complexity theory that has performed a useful role in enterprise ecosystem theory has been that offered by Kauffman regarding the rate of evolution of novelty is that as the economic fabric of the ecosystems within which they are embedded themselves evolve and become more complex in terms of their power laws of scale and scope, the greater is the opportunity for further, accelerating novelty, including that of enterprises. This connects back to our reference to what once seemed a utopian idea which - as a by-product - could cause a huge release of social energy into enterprise, namely the idea of a "basic income". Sufficient thought is now being given at national and regional governmental levels that it is beginning to be tentatively applied in practice for the first time since the Levellers and Diggers.

\section{HIGHLY INFLUENTIAL ARTICLE}

We used the following article as a basis of our evaluation:

Cooke, P. (2016). The virtues of variety in regional innovation systems and entrepreneurial ecosystems. Journal of Open Innovation: Technology, Market, and Complexity, 2(1).

This is the link to the publisher's website:

https://www.sciencedirect.com/science/article/pii/S0048733318301987

\section{INTRODUCTION}

This paper presentation looks back on an effort to counterbalance the growing emergence of neoliberal hegemony in regional political economy at the beginning of the present millennium. In a paper by Cooke (2002) which existed as a guide for EU policy makers to an alternative approach to "new growth theory" and especially that variant that stressed "endogenous growth theory" (Romer 1990) the idea of "generative growth" was formed. This derived from very different and equally deep theoretical roots to those of the neoclassical perspective. Basically, it was critical of the radical individualisn, determinism and linearity of the neoclassical perspective and preferred an evolutionary, socially interactive and non-linear approach to political economy. Of course, history reveals that zero attention was paid to my advice by the relevant EU policy maker team. They followed the various Goldman Sachs, not to say Lehman Brothers, herds like their US policy mentors had approved. This included bending over backwards to allow US corporate high-tech technology commercialisers to retain transfer-priced tax, health service and other public contracts, and free trade (TTIP) benefits to accrue to their rivalrous competitors. Most shocking was the way corporates and the US government first warned then cowed the EU with legal threats against "competitive advantage" in their own backyards (Myant 2015). The proposed trade agreement would fuel concerns that, for example, sovereign UK National Health Service trusts could come under attack from private contractors using the ISDS (investor state dispute settlement) tribunal system. This simulates a kind of "Economic Caesarism" reminiscent of appropriation rule in Roman Britain for the contemporary era of global competitiveness by all possible means.

In reconsidering the massive growth of neoclassical ideology and the even greater growth in malfunctioning of social relations, catastrophic effects of war, refugees and migration that have beset the global economic space of regions, nations and broad territories, the first thing to record has been the decline of co-operative, socially interactive and collaborative action in economic affairs more generally from 2000- 
2016. This may be displaying growing signs of decay but the power of capital's vice-like grip on the leading institutions of the global, bloc and national economies has continued to strengthen. Where may signs of the emergence of more evolutionary, socially responsive and learning predispositions be found? Amongst a few may be counted the continued growth, albeit not overwhelming, of collective innovation and entrepreneurship activity. Both depend significantly on knowledge formation, exploration, examination and exploitation among "strange attractors" who are often capable of interacting across borders (industries, sectors, clusters) to create and innovate with different kinds of creative actors. Although they may compete, they are also capable of collaborating to create something of commercialisable social value. Furthermore, while narrowly-defined science, technology innovation STI patent data is carefully recorded, the much greater contribution to innovative value from DUI "doing, using interaction" activity is widely acknowledged to be under or even un-recorded in the same way. It is like "dark material" in the universe, all around us but difficult to see. Entrepreneurs and innovators are largely responsible for such "dark material".

\section{CONCLUSION}

We have reached the three main discussion points of the foregoing analysis, as a prelude to the three concluding points to which they are related. The first of these opens up a more holistic, integrated and communal mode of institutional economic practice. This privileges - to an extent - the individualistic profit-seeking entrepreneur, though it prefers the more neutral and various characteristics of the enterprise as the vehicle for value analysis and realisation. This correlates with the notion of "generative growth", a more evolutionary, communal and socially interactive learning process than the narrower theory of endogenous growth. This is reductionist as well as individualistic and determined by its main focus on the profit motive par excellence. However "endogenous growth" is no greater insurance than "off the shelf" technology purchase, which is largely at the whim of the entrepreneur's risk judgement. By contrast, "generative growth" collectively exploits the strength in numbers that a more disinterested mode of business practice, gaining from "club goods" and "risk spreading" as described for classic Marshallian industrial districts. This resonates with the more communal, interactive learning disposition of regional innovation systems. These are even more disinterested in their innovations than entrepreneurs are in their profits. This is because they are driven by a problem-solving or discovery methodology that may not, even in the longrun, dependent upon context, reach profitability. To survive, they may have to construct new business models that have the character of "knowledge insurance" strategies (eg biotechnology start-ups; at a corporate scale, price-fixing by the state performs a comparable function towards nuclear energy generation). Just as entrepreneurial/enterprise ecosystems and regional innovation systems may have good and bad exemplars of acceptable practice. That associated with entrepreneurial ecosystems is much closer to the market in purpose, where for innovation systems sub-optimal performance is inclined to act "with good will" but be betrayed by "asymmetric information", adverse selection" or "market failure" issues that may blow policy off course (Stam 2015).

The second discussion point is the extent to which the enterprise ecosystem has yet developed, conceptually, let alone in terms of real practice, capabilities in relation to one of the key concepts in this paper, as expressed in its title, namely to develop a research methodology to handle diversity or, specifically, in the context of this paper "related variety" (including "revealed related variety"). This would entail multiple research designs, themselves diverse, to trace enterprise mutations or enterprise biographies that reveal themselves not as undifferentiated profit taking enterprises but purposive economic entities. These would construct their purposive actions in relation to other enterprises that facilitated the achievement of the higher purpose, such as the (LED) lightbulb, the smartphone or the prosthetic hip or knee joint. There is relatively little of this "ancestry analysis" by comparison with the volumes of studies of "the world's oldest firm" in entrepreneurship studies. There may be some risk attached to formulating "industry interface" or "cross-over" inter- sector or inter-cluster studies but from an innovation standpoint much innovation has this "related variety" character including "revealed" value occurring by accident as much as design. However it is the purposiveness that justifies the risk for search and selection potential. 
And as a final discussion point, in regard to our discussion of statics and dynamics in relation to enterprise ecosystems. It seems likely that the more evolutionary ecosystem perspective may, if tackled and solved methodologically, assist the emergence of a more dynamic profiling of enterprise management if not entrepreneurship in itself. Static equilibrium and making safe bets by the inclination to imitate or "swarm" in Schumpeterian terms rather than engage in true risk-taking practice by backing purposive innovation for social value is holding back study in the field. Armed with the creativity implicit in crossover and other kinds of entrepreneurial/enterprise discovery (EDP) enterprise becomes more dynamic because embedded in emergent and evolving ecosystems. These are structured where feasible into clusterplatforms that celebrate their revealed related variety, for example engaging oil companies in conversations with food firms over biofuel energy, or watchmakers and combustion engine producers finding joint new markets and seat fabric users of nanotechnology to evolve new materials for antiseptic medical uniforms. The creative enterprise will be alive to such (unpredictable) revealed related variety outcomes because it celebrates dynamic change.

In terms of main conclusions, one of the strongest, theoretically and in analytical practice is that if entrepreneurship is difficult and enterprise management is circumscribed by the problem of "satisficing" and "bounded rationality," enterprise ecosystems are advantaged by their networking propensity, their regional "communities of practice" and the free goods of interactive learning from ecosystem peers whether firms or intermediaries. Thus although it is an immature field, arising from "booster practice" rather than well-argued researcher discovery and real-world practical observation, it has worthwhile potential for becoming a dynamic contributor to theory and policy. Importantly, it is by now clear that entrepreneurship is different in nature and kind from innovative activity. But it is also clear that much effort conducted in enterprise ecosystems will be less than high growth firm performance based on hitting the STI jackpot. Rather it will be solid, necessary, problem-solving along the more normal DUI innovation routeways which account for the greater part of social value arising from the enerprise ecosystem setting.

Second, one of the insights of evolutionary complexity theory (ECT) that has performed a useful role in enterprise ecosystem theory has been that offered by Kauffman (2008) regarding the rate of evolution of novelty (innovation or creative artifact or service) is that as the economic fabric of the ecosystems within which they are embedded themselves evolve and become more complex in terms of their power laws of scale and scope, the greater is the opportunity for further, accelerating novelty, including that of enterprises. This connects back to our reference to what once seemed a utopian idea which - as a by-product - could cause a huge release of social energy into enterprise, namely the idea of a "basic income". Sufficient thought is now being given at national and regional governmental levels that it is beginning to be tentatively applied in practice for the first time since the Levellers and Diggers. Such is the vast burden of public expenditure occasioned by the huge weight of healthcare and welfare services and the armies of public servants that administer what can amount to $40 \%+$ of national GDP that conditions are becoming appropriate to consider "basic income" for all citizens. Keep in mind the proposed Swiss monthly stipend is $£ 1,700(\$ 2,400)$ paid to every citizen, a rate of $£ 20,400(\$ 28,000)$. The Swiss health and welfare (including administration) budget Adult citizen population is 4.8 million. Swiss GDP in 2016 was $\$ 475$ billion (PPP); Health and Welfare GDP is $36 \%$. Thus approximately $\$ 171$ billion of GDP is spent on Health and Welfare. This compares with $\$ 140$ billion in proposed Basic Income, a rough saving per annum of $\$ 31$ billion to Swiss taxpayers. Some portion of this is private expenditure but even so, with two-thirds devoted to social security, public expenditure is substantial. The point here is that a surge of enterprise formation could be expected from these Health and Welfare ecosystems, which can be expected to be dynamic, interactive, efficient and effective enterprises despite inevitable start-up and even maturer failures.

Finally, "variety" especially "related variety" (including "revealed related variety") in respect of "enterprise ecosystems" having been specified in terms of search, selection and structuring of enterprise potential in relation to ecosystem opportunities, offers a new and more "path-creating" trajectory of new and diversified business opportunity than more "path dependent" ways of thinking about entrepreneurship. In relation to the separate and distinctive but innovation-directed systems with which future, structured enterprise ecosystems (EE) may come to interact, the RIS and EE joint model offers a powerful conceptual and practical framework for purposive action. In "grand challenge" contexts like "ageing", "healthcare 
surges" and "ambient assisted living" each of which place enormous burdens on taxpayer resources and public investment, there could be no better global scale experiment upon which to begin evolving tools for complexity in both RIS and EE. Accordingly, with the latter even closer to the patient-provider interface than the RIS perspective, the interesting idea of "enterprise ecosystems" or, if preferred "entrepreneurial ecosystems" further underlines the potential of new opportunities for developing not only the "virtues" but the "value of variety".

\section{REFERENCES}

Andersen, E. (2011). Schumpeter and regional innovation. In P. Cooke, B. Asheim, R. Boschma, R. Martin, D. Schwartz, \& F. Tödtling (Eds.), Handbook of regional innovation \& growth. Cheltenham: Edward Elgar.

Arthur, B. (1994). Increasing Returns and Path Dependence in the Economy. Ann Arbor, Michigan University Press.

Arthur, B. (2009). The nature of technology. London: Penguin.

Balconi, M., Brusoni, S., \& Orsenigo, L. (2010). In defence of the linear model: an essay. Research Policy, 39, 1-13.

Boisot, M., Nordberg, M., Yami, S., \& Nicquevert, B. (2011). Collisions \& collaboration: The organization of learning in the atlas experiment in the LHC. Oxford: Oxford University Press.

Boschma, R., \& Martin, R. (2010). The handbook of evolutionary economic geography. Cheltenham: Edward Elgar.

Casson, M. (2003). The entrepreneur: An economic theory (2nd edition). Cheltenham: Edward Elgar.

Cooke, P. (2002). Generative growth, knowledge economies and sustainable development: Implications for regional foresight policy (Paper prepared for 'mobilising the potential of regional foresight actors for an enlarged). Brussels: EU' STRATA-ETAN Expert Group Action and Conference.

Cooke, P. (2012). Complex adaptive innovation systems. London: Routledge.

Cooke, P. (2013). Qualitative analysis and comparison of firm and system incumbents in the New ICT global innovation network. European Planning Studies, 21, 1-18.

Cooke, P. (2015). Boosting skills ecosystems for greener jobs. Paris: OECD.

Cooke, P. (Ed.). (1995). The rise of the Rustbelt. London: UCL Press.

Cooke, P., Boekholt, P., \& Tödtling, F. (2000). The Governance of Innovation in Europe. London: Pinter.

Cooke, P., Heidenreich, M., \& Braczyk, H. (Eds.). (2004). Regional innovation systems (2nd ed.). London: Routledge.

Dosi, G. (1982). Technological paradigms and technological trajectories: a suggested interpretation of the determinants and directions of technological change. Research Policy, 11, 147-162.

Dunning, J. (Ed.). (2000). Regions, globalization, and the knowledge-based economy. Oxford: Oxford University Press.

European Commission. (2012). Guide to research and innovation strategies for smart specialisation. Brussels: European Commission.

Fearn, H. (2016, March 9). Is the citizen's basic income an idea whose time has finally come? The Independent, p. 29.

Freeman, C., \& Perez, C. (1988). Structural crisis of adjustment, business cycles and investment behaviour. In G. Dosi (Ed.), Technical change \& economic theory. London: Pinter.

Green, P. (1983). Considerations on the democratic division of labour. Politics \& Society, 12(4), 445485.

Green, P. (1985). Retrieving democracy: In search of civic equality. Totoma: Rowman \& Allenheld.

Hirst, P., \& Thompson, P. (1996). The globalization question. Cambridge: Polity.

Kauffman, S. (2008). Reinventing the sacred. New York: Basic Books.

Kenney, M. (1986). Schumpeterian innovation and entrepreneurs in capitalism: A case study of the US biotechnology industry. Research Policy, 15, 21-31.

Kirzner, I. (1973). Competition and entrepreneurship. Chicago: University of Chicago Press. 
Kirzner, I. (1997). Entrepreneurial Discovery and the Competitive Market Process: An Austrian Approach, Journal of Economic Literature, 35(1), 60-85.

Kleisch, E., Sloan, A., \& Melvin, E. (2017). Using a Faculty Training and Development Model to Prepare Faculty to Facilitate an Adaptive Learning Online Classroom Designed for Adult Learners. Journal of Higher Education Theory and Practice, 17(7). Retrieved from https://articlegateway.com/index.php/JHETP/article/view/1470

Knight, F. (1921). Risk, uncertainty and profit. Boston: Hart, Schaffner \& Marx.

Krugman, P. (2000). Where in the world is the 'New economic Geography'? In G. Clark, M. Feldman, \& M. Gertler (Eds.), The Oxford handbook of economic geography. Oxford: Oxford University Press.

Lukes, S. (1974). Power: A Radical View. London: Macmillan.

Malecki, E. (2011). Connecting local entrepreneurial ecosystems to global innovation networks. International Journal of Entrepreneurship \& Innovation Management, 14, 36-59.

March, J. (1991). Exploration and exploitation in organisational learning. Organisation Sciences, 2, 7187.

Marshall, A. (1890). Industry \& Trade. London, Macmillan.

Myant, M. (2015). TTIP: What it will mean for us and what is the alternative? Briefing Paper No. 11. Brussels: Observatoire Social Europeén.

Romer, P. (1990). Endogenous technical change. Journal of Political Economy, 98(No. 5, Part 2), S71S102.

Ruigrok, W., \& Van Tulder, R. (1995). The logic of international restructuring. London: Routledge.

Saxenian, A. (1994). Regional advantage. Harvard: Harvard University Press.

Schultz, T. (1982). Investment in entrepreneurial ability. Scandinavian Journal of Economics, 82(4), 43748.

Schumpeter, J. (1932/2005). Development. Journal of Economic Literature, XLIII, 104-116

Schumpeter, J. (1934). The theory of economic development. New Brunswick: Transaction Books.

Schumpeter, J. (1975). Capitalism, Socialism \& Democracy. New York, Harper Torchbooks.

Stacey. (2002). Strategic management \& organisational dynamics: The challenge of complexity. Harlow: Prentice Hall.

Stam, E. (2007). Entrepreneurship and innovation policy. Delft: European Association of Evolutionary Political Economy.

Stam, E. (2015). Entrepreneurial ecosystems and regional policy: A sympathetic critique. European Planning Studies, 23(9), 1759-1769.

Sternberg, R. (2007). Entrepreneurship, proximity and regional innovation systems. Tijdschrift voor Economische en Sociale Geografie, 98(5), 652-666.

Wassermann, P., Schiller, D., \& Thomsen, S. (2016). Spatial co-operation patterns and their impact on innovation outcomes: lessons from firms in a low-technology region. European Planning Studies, 24(5), 1-3.

\section{TRANSLATED VERSION: SPANISH}

Below is a rough translation of the insights presented above. This was done to give a general understanding of the ideas presented in the paper. Please excuse any grammatical mistakes and do not hold the original authors responsible for these mistakes.

\section{VERSION TRADUCIDA: ESPAÑOL}

A continuación se muestra una traducción aproximada de las ideas presentadas anteriormente. Esto se hizo para dar una comprensión general de las ideas presentadas en el documento. Por favor, disculpe cualquier error gramatical y no responsabilite a los autores originales de estos errores. 


\section{INTRODUCCIÓN}

Esta presentación de trabajo mira hacia atrás en un esfuerzo por contrarrestar el creciente surgimiento de la hegemonía neoliberal en la economía política regional a principios del presente milenio. En un artículo de Cooke (2002) que existía como una guía para los responsables políticos de la UE a un enfoque alternativo a la "nueva teoría del crecimiento" y especialmente esa variante que enfatizaba la "teoría del crecimiento endógeno" (Romer 1990) se formó la idea del "crecimiento generativo". Esto derivó de raíces teóricas muy diferentes e igualmente profundas a las de la perspectiva neoclásica. Básicamente, criticaba la individualidad radical, el determinismo y la linealidad de la perspectiva neoclásica y prefería un enfoque evolutivo, socialmente interactivo y no lineal de la economía política. Por supuesto, la historia revela que el equipo responsable de las políticas pertinentes de la UE no prestó ninguna atención a mi consejo. Siguieron a los diversos rebaños de Goldman Sachs, por no decir Lehman Brothers, como sus mentores de política estadounidense habían aprobado. Esto incluyó inclinarse hacia atrás para permitir que los comercializadores corporativos de tecnología de alta tecnología de los Estados Unidos retengan los impuestos a los precios de transferencia, los servicios de salud y otros contratos públicos, y los beneficios del libre comercio (TTIP) se acumulen a sus competidores rivales. Lo más impactante fue la forma en que las empresas y el gobierno de los Estados Unidos primero advirtieron y luego intimidaron a la UE con amenazas legales contra la "ventaja competitiva" en sus propios patios traseros (Myant 2015). El acuerdo comercial propuesto alimentaría las preocupaciones de que, por ejemplo, los fideicomisos soberanos del Servicio Nacional de Salud del Reino Unido podrían ser atacados por contratistas privados que utilizan el sistema de tribunales ISDS (solución de controversias entre inversores y estados). Esto simula una especie de "cesarismo económico" que recuerda a la regla de apropiación en la Gran Bretaña romana para la era contemporánea de la competitividad global por todos los medios posibles.

Al reconsiderar el crecimiento masivo de la ideología neoclásica y el crecimiento aún mayor en el mal funcionamiento de las relaciones sociales, los efectos catastróficos de la guerra, los refugiados y la migración que han acosado el espacio económico global de regiones, naciones y territorios amplios, lo primero que se ha registrado ha sido el declive de la acción cooperativa, socialmente interactiva y colaborativa en asuntos económicos en general de 2000 a 2016. Esto puede estar mostrando signos crecientes de decadencia, pero el poder del control vicioso del capital sobre las principales instituciones de las economías globales, de bloques y nacionales ha seguido fortaleciéndose. ¿Dónde pueden encontrarse signos de la aparición de predisposiciones más evolutivas, socialmente receptivas y de aprendizaje? Entre unos pocos se puede contar el crecimiento continuo, aunque no abrumador, de la innovación colectiva y la actividad empresarial. Ambos dependen significativamente de la formación, exploración, examen y explotación del conocimiento entre "atractores extraños" que a menudo son capaces de interactuar a través de las fronteras (industrias, sectores, clústeres) para crear e innovar con diferentes tipos de actores creativos. Aunque pueden competir, también son capaces de colaborar para crear algo de valor social comercializable. Además, si bien los datos de patentes de ITS de innovación científica y tecnológica estrechamente definidos se registran cuidadosamente, la contribución mucho mayor al valor innovador de la actividad de DUI de "hacer, usar interacción" es ampliamente reconocida como inferior o incluso no registrada de la misma manera. Es como el "material oscuro" en el universo, a nuestro alrededor pero difícil de ver. Los empresarios e innovadores son en gran parte responsables de este "material oscuro".

\section{CONCLUSIÓN}

Hemos llegado a los tres principales puntos de discusión del análisis anterior, como preludio de los tres puntos finales a los que están relacionados. El primero de ellos abre un modo más holístico, integrado y comunitario de práctica económica institucional. Esto privilegia, hasta cierto punto, al empresario individualista que busca ganancias, aunque prefiere las características más neutrales y diversas de la empresa como vehículo para el análisis y la realización del valor. Esto se correlaciona con la noción de "crecimiento generativo", un proceso de aprendizaje más evolutivo, comunitario y socialmente interactivo que la teoría más estrecha del crecimiento endógeno. Esto es reduccionista, así como individualista y 
determinado por su enfoque principal en el afán de lucro por excelencia. Sin embargo, el "crecimiento endógeno" no es un seguro mayor que la compra de tecnología "lista para usar", que en gran medida es por el capricho del juicio de riesgo del empresario. Por el contrario, el "crecimiento generativo" explota colectivamente la fuerza en números que un modo más desinteresado de práctica comercial, ganando de los "bienes del club" y la "propagación del riesgo" como se describe para los distritos industriales clásicos de Marshall. Esto resuena con la disposición de aprendizaje más comunitaria e interactiva de los sistemas regionales de innovación. Estos están aún más desinteresados en sus innovaciones que los empresarios en sus ganancias. Esto se debe a que están impulsados por una metodología de resolución de problemas o descubrimiento que, incluso a largo plazo, dependiendo del contexto, puede alcanzar la rentabilidad. Para sobrevivir, es posible que tengan que construir nuevos modelos de negocio que tengan el carácter de estrategias de "seguro del conocimiento" (por ejemplo, nuevas empresas de biotecnología; a escala corporativa, la fijación de precios por parte del estado realiza una función comparable hacia la generación de energía nuclear). Del mismo modo que los ecosistemas empresariales y los sistemas regionales de innovación pueden tener buenos y malos ejemplos de prácticas aceptables. El asociado con los ecosistemas empresariales está mucho más cerca del mercado en propósito, donde para los sistemas de innovación el rendimiento subóptimo se inclina a actuar "con buena voluntad" pero ser traicionado por "información asimétrica", selección adversa" o "falla del mercado" cuestiones que pueden desviar la política del curso (Stam 2015).

El segundo punto de discusión es la medida en que el ecosistema empresarial aún ha desarrollado, conceptualmente, y mucho menos en términos de práctica real, capacidades en relación con uno de los conceptos clave en este documento, como se expresa en su título, a saber, desarrollar una metodología de investigación para manejar la diversidad o, específicamente, en el contexto de este documento "variedad relacionada" (incluida la "variedad relacionada revelada"). Esto implicaría múltiples diseños de investigación, en sí mismos diversos, para rastrear mutaciones empresariales o biografías empresariales que se revelan no como empresas con ganancias indiferenciadas, sino como entidades económicas intencionales. Estos construirían sus acciones intencionales en relación con otras empresas que facilitaran el logro del propósito superior, como la bombilla (LED), el teléfono inteligente o la prótesis de cadera o articulación de la rodilla. Hay relativamente poco de este "análisis de ascendencia" en comparación con los volúmenes de estudios de "la empresa más antigua del mundo" en estudios de emprendimiento. Puede haber algún riesgo asociado a la formulación de estudios intersectoriales o intergrupistas de "interfaz de la industria" o "cruzados", pero desde el punto de vista de la innovación, gran parte de la innovación tiene este carácter de "variedad relacionada", incluido el valor "revelado" que ocurre por accidente tanto como el diseño. Sin embargo, es la intencionalidad la que justifica el riesgo para el potencial de búsqueda y selección.

Y como punto de discusión final, con respecto a nuestra discusión de la estática y la dinámica en relación con los ecosistemas empresariales. Parece probable que la perspectiva más evolutiva del ecosistema pueda, si se aborda y resuelve metodológicamente, ayudar a la aparición de un perfil más dinámico de la gestión empresarial, si no el espíritu empresarial en sí mismo. El equilibrio estático y hacer apuestas seguras por la inclinación a imitar o "enjambre" en términos schumpeterianos en lugar de participar en una verdadera práctica de toma de riesgos al respaldar la innovación intencional para el valor social está frenando el estudio en el campo. Armada con la creatividad implícita en el cross-over y otros tipos de descubrimiento empresarial / empresarial (EDP), la empresa se vuelve más dinámica porque está integrada en ecosistemas emergentes y en evolución. Estos se estructuran cuando es factible en plataformas de clúster que celebran su variedad relacionada revelada, por ejemplo, involucrando a las compañías petroleras en conversaciones con empresas de alimentos sobre energía de biocombustibles, o relojeros y productores de motores de combustión que encuentran nuevos mercados conjuntos y colocan a los usuarios de telas de nanotecnología para desarrollar nuevos materiales para uniformes médicos antisépticos. La empresa creativa estará viva a tales (impredecibles) resultados de variedad relacionados revelados porque celebra el cambio dinámico.

En términos de conclusiones principales, una de las más sólidas, teóricamente y en la práctica analítica es que si el espíritu empresarial es difícil y la gestión empresarial está circunscrita por el problema de la 
"satisfacción" y la "racionalidad limitada", los ecosistemas empresariales se ven favorecidos por su propensión a la creación de redes, sus "comunidades de práctica" regionales y los bienes gratuitos del aprendizaje interactivo de sus pares del ecosistema, ya sean empresas o intermediarios. Por lo tanto, aunque es un campo inmaduro, que surge de la "práctica de refuerzo" en lugar del descubrimiento de investigadores bien argumentado y la observación práctica del mundo real, tiene un potencial valioso para convertirse en un contribuyente dinámico a la teoría y la política. Es importante destacar que ya está claro que el espíritu empresarial es diferente en naturaleza y tipo de la actividad innovadora. Pero también está claro que gran parte del esfuerzo realizado en los ecosistemas empresariales será menor que el rendimiento de la empresa de alto crecimiento basado en ganar el premio mayor de LAS ITS. Más bien, será sólido, necesario, de resolución de problemas a lo largo de las rutas de innovación de DUI más normales que representan la mayor parte del valor social que surge del entorno del ecosistema enerprise.

En segundo lugar, una de las ideas de la teoría de la complejidad evolutiva (ECT) que ha desempeñado un papel útil en la teoría de los ecosistemas empresariales ha sido la ofrecida por Kauffman (2008) con respecto a la tasa de evolución de la novedad (innovación o artefacto creativo o servicio) es que a medida que el tejido económico de los ecosistemas en los que están incrustados evolucionan y se vuelven más complejos en términos de sus leyes de poder de escala y alcance, cuanto mayor es la oportunidad de acelerar aún más la novedad, incluida la de las empresas. Esto se conecta con nuestra referencia a lo que una vez pareció una idea utópica que, como subproducto, podría causar una gran liberación de energía social en la empresa, a saber, la idea de una "renta básica". Ahora se está pensando lo suficiente a nivel gubernamental nacional y regional que está comenzando a aplicarse tentativamente en la práctica por primera vez desde los Levellers y Diggers. Tal es la inmensa carga del gasto público ocasionada por el enorme peso de los servicios sanitarios y asistenciales y los ejércitos de funcionarios públicos que administran lo que puede ascender al $40 \%+$ del PIB nacional que se están volviendo adecuadas las condiciones para considerar la "renta básica" para todos los ciudadanos. Tenga en cuenta que el estipendio mensual suizo propuesto es de $£ 1,700$ (\$2,400) pagado a cada ciudadano, una tasa de $£ 20,400(\$ 28,000)$. El presupuesto suizo de salud y bienestar (incluida la administración) La población de ciudadanos adultos es de 4,8 millones. El PIB suizo en 2016 fue de $\$ 475$ mil millones (PPA); El PIB de Salud y Bienestar es del $36 \%$. Por lo tanto, aproximadamente \$ 171 mil millones del PIB se gastan en Salud y Bienestar. Esto se compara con \$ 140 mil millones en la Renta Básica propuesta, un ahorro aproximado por año de \$ 31 mil millones para los contribuyentes suizos. Una parte de esto es gasto privado, pero aun así, con dos tercios dedicados a la seguridad social, el gasto público es sustancial. El punto aquí es que se podría esperar un aumento de la formación de empresas de estos ecosistemas de salud y bienestar, que se puede esperar que sean empresas dinámicas, interactivas, eficientes y efectivas a pesar de las inevitables fallas iniciales e incluso maduras.

Por último, la "variedad", especialmente la "variedad relacionada" (incluida la "variedad relacionada revelada") con respecto a los "ecosistemas empresariales", que se ha especificado en términos de búsqueda, selección y estructuración del potencial empresarial en relación con las oportunidades de los ecosistemas, ofrece una trayectoria nueva y más "creadora de caminos" de oportunidades de negocio nuevas y diversificadas que formas de pensar más "dependientes del camino" sobre el espíritu empresarial. En relación con los sistemas separados y distintivos, pero dirigidos a la innovación, con los que los futuros ecosistemas empresariales estructurados (EE) pueden llegar a interactuar, el modelo conjunto RIS y EE ofrece un poderoso marco conceptual y práctico para la acción intencional. En contextos de "gran desafío" como el "envejecimiento", las "oleadas de atención médica" y la "vida asistida por el medio ambiente", cada una de las cuales impone enormes cargas a los recursos de los contribuyentes y la inversión pública, no podría haber un mejor experimento a escala global sobre el cual comenzar a desarrollar herramientas para la complejidad tanto en RIS como en EE. En consecuencia, con este último aún más cerca de la interfaz paciente-proveedor que la perspectiva RIS, la interesante idea de "ecosistemas empresariales" o, si se prefiere, "ecosistemas empresariales" subraya aún más el potencial de nuevas oportunidades para desarrollar no solo las "virtudes" sino el "valor de la variedad". 


\section{TRANSLATED VERSION: FRENCH}

Below is a rough translation of the insights presented above. This was done to give a general understanding of the ideas presented in the paper. Please excuse any grammatical mistakes and do not hold the original authors responsible for these mistakes.

\section{VERSION TRADUITE: FRANÇAIS}

Voici une traduction approximative des idées présentées ci-dessus. Cela a été fait pour donner une compréhension générale des idées présentées dans le document. Veuillez excuser toutes les erreurs grammaticales et ne pas tenir les auteurs originaux responsables de ces erreurs.

\section{INTRODUCTION}

Cette présentation de l'article revient sur un effort visant à contrebalancer l'émergence croissante de l'hégémonie néolibérale dans l'économie politique régionale au début du présent millénaire. Dans un article de Cooke (2002) qui existait comme guide pour les décideurs politiques de l'UE vers une approche alternative de la "nouvelle théorie de la croissance » et en particulier cette variante qui soulignait la « théorie de la croissance endogène » (Romer 1990), l'idée de « croissance générative » a été formée. Cela découlait de racines théoriques très différentes et tout aussi profondes que celles de la perspective néoclassique. Fondamentalement, il critiquait l'individualité radicale, le déterminisme et la linéarité de la perspective néoclassique et préférait une approche évolutionniste, socialement interactive et non linéaire de l'économie politique. Bien sûr, l'histoire révèle qu'aucune attention n'a été accordée à mes conseils par l'équipe compétente des décideurs politiques de l'UE. Ils ont suivi les différents groupes goldman Sachs, pour ne pas dire Lehman Brothers, comme leurs mentors politiques américains l'avaient approuvé. Cela comprenait le fait de se plier en quatre pour permettre aux entreprises américaines de commercialiser des technologies de haute technologie de conserver les droits de transfert, les services de santé et autres contrats publics, ainsi que les avantages du libre-échange (TTIP) à leurs concurrents rivaux. Le plus choquant a été la façon dont les entreprises et le gouvernement américain ont d'abord mis en garde puis intimidé l'UE avec des menaces juridiques contre « l'avantage concurrentiel » dans leur propre cour (Myant 2015). L'accord commercial proposé alimenterait les craintes que, par exemple, les fiducies souveraines du National Health Service du Royaume-Uni puissent être attaquées par des entrepreneurs privés utilisant le système judiciaire RDIE (règlement des différends entre investisseurs et États). Cela simule une sorte de « césarisme économique » qui rappelle la règle d'appropriation en Grande-Bretagne romaine pour l'ère contemporaine de la compétitivité mondiale par tous les moyens possibles.

En reconsidérant la croissance massive de l'idéologie néoclassique et la croissance encore plus grande du dysfonctionnement des relations sociales, les effets catastrophiques de la guerre, des réfugiés et des migrations qui ont assailli l'espace économique mondial des régions, des nations et des vastes territoires, la première chose à enregistrer a été le déclin de l'action coopérative, socialement interactive et collaborative dans les affaires économiques plus généralement de 2000 à 2016. Cela montre peut-être des signes croissants de décadence, mais le pouvoir de l'emprise du capital sur les principales institutions des économies mondiales, de bloc et nationales a continué de se renforcer. Où peut-on trouver des signes de l'émergence de prédispositions plus évolutives, socialement réactives et apprenantes ? Parmi quelques-uns, on peut compter la croissance continue, bien que non écrasante, de l'innovation collective et de l'activité entrepreneuriale. Les deux dépendent de manière significative de la formation, de l'exploration, de l'examen et de l'exploitation des connaissances parmi les « attracteurs étranges » qui sont souvent capables d'interagir au-delà des frontières (industries, secteurs, clusters) pour créer et innover avec différents types d'acteurs créatifs. Bien qu'ils puissent être compétitifs, ils sont également capables de collaborer pour créer quelque chose de valeur sociale commercialisable. En outre, bien que les données étroitement définies sur les brevets en matière de science et d'innovation technologique soient soigneusement enregistrées, la contribution beaucoup plus importante à la valeur novatrice de l'activité de DUI «faire, utiliser 
l'interaction » est largement reconnue comme faisant ou même non enregistrée de la même manière. C'est comme une « matière sombre » dans l'univers, tout autour de nous mais difficile à voir. Les entrepreneurs et les innovateurs sont en grande partie responsables de ce «matériau sombre ».

\section{CONCLUSION}

Nous sommes arrivés aux trois principaux points de discussion de l'analyse précédente, en prélude aux trois points de conclusion auxquels ils sont liés. Le premier d'entre eux ouvre un mode de pratique économique institutionnelle plus holistique, intégré et communautaire. Cela privilégie - dans une certaine mesure - l'entrepreneur individualiste à la recherche du profit, bien qu'il préfère les caractéristiques plus neutres et diverses de l'entreprise comme véhicule d'analyse et de réalisation de la valeur. Cela est en corrélation avec la notion de "croissance générative ", un processus d'apprentissage plus évolutif, communautaire et socialement interactif que la théorie plus étroite de la croissance endogène. Ceci est à la fois réductionniste et individualiste et déterminé par son accent principal sur la motivation du profit par excellence. Cependant, la «croissance endogène » n'est pas une plus grande assurance que l'achat de technologies «prêtes à l'emploi », qui est en grande partie au gré du jugement de risque de l'entrepreneur. En revanche, la « croissance génératrice » exploite collectivement la force du nombre qu'un mode de pratique commerciale plus désintéressé, gagnant des « biens de club » et de la " propagation des risques » comme décrit pour les districts industriels marshalliens classiques. Cela résonne avec la disposition d'apprentissage plus communautaire et interactive des systèmes d'innovation régionaux. Ceux-ci sont encore plus désintéressés par leurs innovations que les entrepreneurs ne le sont par leurs profits. En effet, ils sont guidés par une méthodologie de résolution de problèmes ou de découverte qui peut, même à long terme, dépendre du contexte, atteindre la rentabilité. Pour survivre, ils devront peut-être construire de nouveaux modèles d'affaires qui ont le caractère de stratégies d'«assurance de la connaissance » (par exemple, les start-ups biotechnologiques; à l'échelle de l'entreprise, la fixation des prix par l'État remplit une fonction comparable à la production d'énergie nucléaire). Tout comme les écosystèmes d'entreprises et d'entreprises et les systèmes d'innovation régionaux peuvent avoir de bons et de mauvais exemples de pratiques acceptables. Celle associée aux écosystèmes entrepreneuriaux est beaucoup plus proche du marché en termes d'objectif, où, pour les systèmes d'innovation, les performances sous-optimales sont enclines à agir " avec bonne volonté », mais à être trahies par des problèmes d'«information asymétrique », de sélection adverse ou de « défaillance du marché » qui peuvent faire déraxer la politique (Stam 2015).

Le deuxième point de discussion est la mesure dans laquelle l'écosystème des entreprises a encore développé, conceptuellement, sans parler en termes de pratique réelle, des capacités par rapport à l'un des concepts clés du présent document, tel qu'exprimé dans son titre, à savoir développer une méthodologie de recherche pour gérer la diversité ou, plus précisément, dans le contexte de ce document " variété connexe » (y compris «variété connexe révélée »). Cela impliquerait de multiples conceptions de recherche, ellesmêmes diverses, pour retracer les mutations d'entreprise ou les biographies d'entreprises qui se révèlent non pas comme des entreprises de prise de bénéfices indifférenciées, mais comme des entités économiques à dessein. Ceux-ci construiraient leurs actions vis-à-vis d'autres entreprises qui faciliteraient la réalisation de l'objectif supérieur, telles que l'ampoule (LED), le smartphone ou la prothèse de hanche ou d'articulation du genou. Il y a relativement peu de cette « analyse de l'ascendance » par rapport aux volumes d'études de « la plus ancienne entreprise du monde» dans les études sur l'entrepreneuriat. Il peut y avoir un certain risque à formuler des études intersectorielles ou intersectorielles ou intergroupes " croisées », mais du point de vue de l'innovation, une grande partie de l'innovation a ce caractère de " variété connexe », y compris la valeur « révélée » qui se produit par accident autant que par conception. Cependant, c'est l'objectif qui justifie le risque de potentiel de recherche et de sélection.

Et comme point de discussion final, en ce qui concerne notre discussion sur la statique et la dynamique en relation avec les écosystèmes d'entreprise. Il semble probable que la perspective plus évolutive de l'écosystème puisse, si elle est abordée et résolue méthodologiquement, contribuer à l'émergence d'un profilage plus dynamique de la gestion d'entreprise, sinon de l'entrepreneuriat en soi. L'équilibre statique et la mise en valeur sûre par l'inclination à imiter ou à « essaimer » en termes schumpétériens plutôt que de 
s'engager dans une véritable pratique de prise de risque en soutenant l'innovation à dessein pour la valeur sociale freinent les études sur le terrain. Armée de la créativité implicite dans le cross-over et d'autres types d'entrepreneuriat / découverte d'entreprise (EDP), l'entreprise devient plus dynamique parce qu'elle est intégrée dans des écosystèmes émergents et en évolution. Ceux-ci sont structurés lorsque cela est possible en plates-formes de clusters qui célèbrent leur variété connexe révélée, par exemple en engageant les compagnies pétrolières dans des conversations avec des entreprises alimentaires sur l'énergie des biocarburants, ou en des horlogers et des producteurs de moteurs à combustion trouvant de nouveaux marchés communs et des utilisateurs de tissus de siège de nanotechnologie pour faire évoluer de nouveaux matériaux pour les uniformes médicaux antiseptiques. L'entreprise créative sera vivante à ces résultats de variété (imprévisibles) révélés parce qu'elle célèbre le changement dynamique.

En termes de conclusions principales, l'une des plus fortes, théoriquement et dans la pratique analytique, est que si l'entrepreneuriat est difficile et que la gestion d'entreprise est circonscrite par le problème de la " rationalité satisfaisante » et « limitée », les écosystèmes d'entreprise sont avantagés par leur propension à la mise en réseau, leurs « communautés de pratique » régionales et les biens gratuits de l'apprentissage interactif des pairs de l'écosystème, qu'il s'agisse d'entreprises ou d'intermédiaires. Ainsi, bien qu'il s'agit d'un domaine immature, découlant de la « pratique de rappel » plutôt que de la découverte bien argumentée des chercheurs et de l'observation pratique du monde réel, il a un potentiel intéressant pour devenir un contributeur dynamique à la théorie et à la politique. Il est important de savoir qu'il est maintenant clair que l'entrepreneuriat est de nature et de nature différentes de l'activité innovante. Mais il est également clair que beaucoup d'efforts déployés dans les écosystèmes d'entreprise seront inférieurs à la performance des entreprises à forte croissance en fonction de l'atteinte du jackpot STI. Il s'agira plutôt d'une résolution de problèmes solide et nécessaire le long des voies d'innovation DUI plus normales qui représentent la plus grande partie de la valeur sociale découlant du cadre de l'écosystème enerprise.

Deuxièmement, l'une des idées de la théorie de la complexité évolutive (ECT) qui a joué un rôle utile dans la théorie des écosystèmes d'entreprise a été celle offerte par Kauffman (2008) concernant le taux d'évolution de la nouveauté (innovation ou artefact ou service créatif) est qu'à mesure que le tissu économique des écosystèmes dans lesquels ils sont intégrés évolue et devient plus complexe en termes de lois de puissance d'échelle et de portée, la plus grande est la possibilité d'accélérer davantage la nouveauté, y compris celle des entreprises. Cela nous ramène à notre référence à ce qui semblait autrefois une idée utopique qui, en tant que sous-produit, pourrait provoquer une énorme libération d'énergie sociale dans l'entreprise, à savoir l'idée d'un « revenu de base ». Les gouvernements nationaux et régionaux réfléchissent suffisamment au moment où il commence à être provisoirement appliqué dans la pratique pour la première fois depuis les Levellers et les Diggers. L'énorme fardeau des dépenses publiques occasionné par l'énorme poids des services de santé et de protection sociale et des armées de fonctionnaires qui administrent ce qui peut représenter plus de $40 \%$ du PIB national est tel que les conditions deviennent appropriées pour envisager un « revenu de base » pour tous les citoyens. Gardez à l'esprit que l'allocation mensuelle suisse proposée est de $1700 £$ ( 2400 \$) versée à chaque citoyen, soit un taux de $20400 £(28$ $000 \$$ ). Le budget suisse de la santé et de la protection sociale (y compris l'administration) La population citoyenne adulte est de 4,8 millions. En 2016, le PIB suisse s'est situé à 475 milliards de dollars (PPA); Le PIB de la santé et du bien-être est de $36 \%$. Ainsi, environ 171 milliards de dollars du PIB sont consacrés à la santé et au bien-être. Cela se compare à 140 milliards de dollars de revenu de base proposé, soit une économie approximative par an de 31 milliards de dollars pour les contribuables suisses. Une partie de cette dépense est consacrée aux dépenses privées, mais même ainsi, avec les deux tiers consacrés à la sécurité sociale, les dépenses publiques sont substantielles. Le fait est qu'on pourrait s'attendre à une poussée de la création d'entreprises de la part de ces écosystèmes de santé et de bien-être, qui peuvent être considérés comme des entreprises dynamiques, interactives, efficientes et efficaces malgré des démarrages inévitables et des échecs encore plus matures.

Enfin, la « variété », en particulier la " variété connexe » (y compris la « variété connexe révélée ») en ce qui concerne les « écosystèmes d'entreprise » ayant été spécifiée en termes de recherche, de sélection et de structuration du potentiel d'entreprise par rapport aux opportunités d'écosystème, offre une trajectoire nouvelle et plus «créatrice » d'opportunités commerciales nouvelles et diversifiées que des façons plus 
« dépendantes » de penser à l'entrepreneuriat. En ce qui concerne les systèmes distincts et distinctifs, mais axés sur l'innovation, avec lesquels les futurs écosystèmes d'entreprise structurés (EE) pourraient interagir, le modèle conjoint RIS et EE offre un cadre conceptuel et pratique puissant pour une action à dessein. Dans des contextes de " grands défis » tels que le « vieillissement », les « poussées de soins de santé » et l'« aide à la vie autonome ambiante », qui imposent chacun un fardeau énorme aux ressources des contribuables et à l'investissement public, il ne pourrait y avoir de meilleure expérience à l'échelle mondiale sur laquelle commencer à faire évoluer des outils de complexité dans les SIF et l'EE. En conséquence, cette dernière pouvant être encore plus proche de l'interface patient-prestataire que de la perspective RIS, l'idée intéressante d'《 écosystèmes d'entreprise » ou, si l'on préfère, d'《 écosystèmes entrepreneuriaux » souligne davantage le potentiel de nouvelles opportunités pour développer non seulement les «vertus » mais aussi la « valeur de la variété ».

\section{TRANSLATED VERSION: GERMAN}

Below is a rough translation of the insights presented above. This was done to give a general understanding of the ideas presented in the paper. Please excuse any grammatical mistakes and do not hold the original authors responsible for these mistakes.

\section{ÜBERSETZTE VERSION: DEUTSCH}

Hier ist eine ungefähre Übersetzung der oben vorgestellten Ideen. Dies wurde getan, um ein allgemeines Verständnis der in dem Dokument vorgestellten Ideen zu vermitteln. Bitte entschuldigen Sie alle grammatikalischen Fehler und machen Sie die ursprünglichen Autoren nicht für diese Fehler verantwortlich.

\section{EINLEITUNG}

Diese Papierpräsentation blickt auf die Bemühungen zurück, die wachsende Entstehung neoliberaler Hegemonie in der regionalen politischen Ökonomie $\mathrm{zu}$ Beginn des gegenwärtigen Jahrtausends auszugleichen. In einem Papier von Cooke (2002), das als Leitfaden für politische Entscheidungsträger in der EU zu einem alternativen Ansatz zur "neuen Wachstumstheorie und insbesondere zu der Variante, die die endogene" Wachstumstheorie betonte (Romer 1990), existierte, wurde die Idee des generativen Wachstums" gebildet. Dies ergab sich aus ganz anderen und ebenso tiefen theoretischen Wurzeln wie die der neoklassischen Perspektive. Grundsätzlich stand sie dem radikalen Individualismus, Determinismus und der Linearität der neoklassischen Perspektive kritisch gegenüber und bevorzugte einen evolutionären, sozial interaktiven und nichtlinearen Ansatz der politischen Ökonomie. Natürlich zeigt die Geschichte, dass meinem Rat vom zuständigen EU-Politikteam keine Aufmerksamkeit geschenkt wurde. Sie folgten den verschiedenen Goldman Sachs, um nicht zu sagen Lehman Brothers, Herden, wie sie ihre USPolitikmentoren genehmigt hatten. Dazu gehörte das Rückwärtsbeugen, um es US-amerikanischen HighTech-Technologie-Kommerzialisierern zu ermöglichen, Transferpreissteuern, Gesundheitsdienstleistungen und andere öffentliche Aufträge sowie Freihandelsvorteile (TTIP) für ihre rivalisierenden Konkurrenten beizubehalten. Am schockierendsten war die Art und Weise, wie Unternehmen und die US-Regierung die EU zuerst warnten und dann die EU mit rechtlichen Drohungen gegen "Wettbewerbsvorteile" in ihren eigenen Hinterhöfen einschüchterten (Myant 2015). Das vorgeschlagene Handelsabkommen würde Bedenken schüren, dass beispielsweise souveräne britische National Health Service Trusts von privaten Auftragnehmern angegriffen werden könnten, die das ISDS-Gerichtssystem (Investor State Dispute Settlement) nutzen. Dies simuliert eine Art "ökonomischen Cäsarismus", der an die Aneignungsherrschaft im römischen Britannien für die heutige Ära der globalen Wettbewerbsfähigkeit mit allen möglichen Mitteln erinnert.

Bei der Überprüfung des massiven Wachstums der neoklassischen Ideologie und des noch stärkeren Wachstums von Fehlfunktionen sozialer Beziehungen, katastrophalen Auswirkungen von Krieg, 
Flüchtlingen und Migration, die den globalen Wirtschaftsraum von Regionen, Nationen und weiten Territorien heimgesucht haben, war das erste, was zu verzeichnen war, der Rückgang des kooperativen, sozial interaktiven und kollaborativen Handelns in wirtschaftlichen Angelegenheiten im Allgemeinen von 2000 bis 2016. Dies mag wachsende Anzeichen des Verfalls zeigen, aber die Macht des lasterhaften Griffs des Kapitals auf die führenden Institutionen der Globalen-, Block- und Volkswirtschaften hat sich weiter verstärkt. Wo können Anzeichen für die Entstehung evolutionärerer, sozial ansprechenderer und lernfreudiger veranlagte Veranlagungen gefunden werden? $\mathrm{Zu}$ den wenigen zählt das anhaltende, wenn auch nicht überwältigende Wachstum kollektiver Innovations- und Unternehmertätigkeit. Beide hängen maßgeblich von der Wissensbildung, -erforschung, -untersuchung und -nutzung unter "seltsamen Attraktoren" ab, die oft in der Lage sind, über Grenzen hinweg (Branchen, Sektoren, Cluster) zu interagieren, um mit verschiedenen Arten von kreativen Akteuren zu schaffen und zu innovieren. Obwohl sie konkurrieren können, sind sie auch in der Lage, zusammenzuarbeiten, um etwas von kommerziellem sozialem Wert zu schaffen. Während eng definierte STI-Patentdaten für Wissenschaft und Technologieinnovation sorgfältig aufgezeichnet werden, wird allgemein anerkannt, dass der viel größere Beitrag zum Innovationswert der DUI-Aktivität "Doing, Using Interaction" auf die gleiche Weise unteroder sogar nicht aufgezeichnet wird. Es ist wie "dunkles Material" im Universum, überall um uns herum, aber schwer zu sehen. Unternehmer und Innovatoren sind maßgeblich für solches "dunkles Material" verantwortlich.

\section{SCHLUSSFOLGERUNG}

Wir haben die drei Hauptdiskussionspunkte der vorstehenden Analyse erreicht, als Auftakt zu den drei abschließenden Punkten, auf die sie sich beziehen. Die erste eröffnet eine ganzheitlichere, integriertere und gemeinschaftlichere Art der institutionellen Wirtschaftspraxis. Dies privilegiert - bis zu einem gewissen Grad - den individualistischen profitorientierten Unternehmer, bevorzugt jedoch die neutraleren und vielfältigeren Merkmale des Unternehmens als Vehikel für die Wertanalyse und -realisierung. Dies korreliert mit dem Begriff des "generativen Wachstums", einem evolutionäreren, gemeinschaftlicheren und sozial interaktiveren Lernprozess als die engere Theorie des endogenen Wachstums. Diese ist sowohl reduktionistisch als auch individualistisch und bestimmt durch ihren Hauptfokus auf das Profitmotiv schlechthin. "endogenes Wachstum" ist jedoch keine größere Versicherung als der Kauf von Technologie "von der Stange", der weitgehend nach Lust und Laune des Risikourteils des Unternehmers erfolgt. Im Gegensatz dazu nutzt "generatives Wachstum" kollektiv die Stärke in Zahlen, die eine uneigennützigere Art der Geschäftspraxis von "Clubgütern" und "Risikostreuung" profitiert, wie sie für klassische MarshallIndustriebezirke beschrieben wird. Dies steht im Einklang mit der eher gemeinschaftlichen, interaktiven Lerndisposition regionaler Innovationssysteme. Diese sind an ihren Innovationen noch mehr desinteressiert als Unternehmer an ihren Gewinnen. Dies liegt daran, dass sie von einer Problemlösungs- oder Entdeckungsmethodik angetrieben werden, die selbst auf lange Sicht, abhängig vom Kontext, möglicherweise keine Rentabilität erreicht. Um zu überleben, müssen sie möglicherweise neue Geschäftsmodelle konstruieren, die den Charakter von "Wissensversicherungsstrategien" haben (z. B. Biotechnologie-Start-ups; im Unternehmensmaßstab erfüllt die Preisfestsetzung durch den Staat eine vergleichbare Funktion gegenüber der Kernenergieerzeugung). Genauso wie unternehmerische Ökosysteme und regionale Innovationssysteme gute und schlechte Beispiele für akzeptable Praktiken haben können. Das, was mit unternehmerischen Ökosystemen verbunden ist, ist viel näher am Markt, wo für Innovationssysteme suboptimale Leistung dazu neigt, "mit gutem Willen" zu handeln, aber durch "asymmetrische Informationen", negative Selektion oder "Marktversagen" -Probleme verraten wird, die die Politik aus der Bahn nehmen können (Stam 2015).

Der zweite Diskussionspunkt ist das Ausmaß, in dem das Unternehmensökosystem noch fähigkeiten entwickelt hat, konzeptionell, geschweige denn in Bezug auf die reale Praxis, Fähigkeiten in Bezug auf eines der Schlüsselkonzepte in diesem Papier, wie es im Titel zum Ausdruck kommt, nämlich eine

Forschungsmethodik zu entwickeln, um mit Vielfalt umzugehen oder, insbesondere im Kontext dieses Papiers "verwandte Vielfalt" (einschließlich "offenbarte verwandte Vielfalt"). Dies würde mehrere 
Forschungsdesigns erfordern, die ihrer selbst vielfältig sind, um Unternehmensmutationen oder Unternehmensbiografien zu verfolgen, die sich nicht als undifferenzierte Gewinnunternehmen, sondern als zweckgebundene wirtschaftliche Einheiten entpuppen. Diese würden ihre zielgerichteten Maßnahmen in Bezug auf andere Unternehmen konstruieren, die die Erreichung des höheren Zwecks erleichtern, wie die (LED-) Glühbirne, das Smartphone oder das prothetische Hüft- oder Kniegelenk. Von dieser "Abstammungsanalyse" gibt es relativ wenig im Vergleich zu den Studienvolumina der "ältesten Firma der Welt" in der Entrepreneurship-Studien. Es kann ein gewisses Risiko mit der Formulierung von "Branchenschnittstellen" oder "Cross-Over" -Studien zwischen Sektoren oder Clustern verbunden sein, aber aus Innovationssicht hat viele Innovationen diesen "verwandten Vielfalt" -Charakter, einschließlich des "aufgedeckten" Wertes, der zufällig auftritt, ebenso wie design. Es ist jedoch die Zweckgenauigkeit, die das Risiko für Such- und Selektionspotenziale rechtfertigt.

Und als letzten Diskussionspunkt in Bezug auf unsere Diskussion von Statik und Dynamik in Bezug auf Unternehmensökosysteme. Es scheint wahrscheinlich, dass die eher evolutionäre Ökosystemperspektive, wenn sie methodisch angegangen und gelöst wird, die Entstehung einer dynamischeren Profilierung des Unternehmensmanagements, wenn nicht des Unternehmertums an sich, unterstützen kann. Statisches Gleichgewicht und sichere Wetten durch die Neigung, in Schumpeterianischen Begriffen zu imitieren oder zu "schwärmen", anstatt sich an einer echten Risikobereitschaftspraxis zu beteiligen, indem sie zweckgerichtete Innovationen für sozialen Wert unterstützen, bremsen das Studium auf diesem Gebiet. Bewaffnet mit der Kreativität, die in Cross-Over und anderen Arten von Entrepreneurial / Enterprise Discovery (EDV) impliziert ist, wird das Unternehmen dynamischer, weil es in aufstrebende und sich entwickelnde Ökosysteme eingebettet ist. Diese sind, wo möglich, in Cluster-Plattformen strukturiert, die ihre offenbarte verwandte Vielfalt feiern, zum Beispiel Ölunternehmen in Gespräche mit Lebensmittelunternehmen über Biokraftstoffenergie einbeziehen, oder Uhrmacher und Verbrennungsmotorenhersteller, die gemeinsame neue Märkte finden und Sitzstoffnutzer der Nanotechnologie verwenden, um neue Materialien für antiseptische medizinische Uniformen zu entwickeln. Das kreative Unternehmen wird für solche (unvorhersehbaren) offenbarten unterschiedlichen Ergebnisse lebendig sein, weil es dynamische Veränderungen feiert.

In Bezug auf die wichtigsten Schlussfolgerungen ist eine der stärksten, theoretisch und in der analytischen Praxis, dass, wenn Unternehmertum schwierig ist und die Unternehmensführung durch das Problem der "befriedigten" und "begrenzten Rationalität" begrenzt wird, Unternehmensökosysteme durch ihre Vernetzungsneigung, ihre regionalen "Praxisgemeinschaften" und die kostenlosen Güter des interaktiven Lernens von Ökosystemkollegen, ob Unternehmen oder Vermittler, begünstigt werden. Obwohl es sich also um ein unreifes Feld handelt, das eher aus der "Booster-Praxis" als aus gut argumentierten Forscherentdeckungen und praktischen Beobachtungen in der realen Welt entsteht, hat es ein lohnendes Potenzial, ein dynamischer Beitrag zu Theorie und Politik zu werden. Wichtig ist, dass inzwischen klar ist, dass sich Unternehmertum in Art und Art von innovationser Tätigkeit unterscheidet. Aber es ist auch klar, dass viele Anstrengungen, die in Unternehmensökosystemen unternommen werden, weniger sein werden als die Leistung von Unternehmen mit hohem Wachstum, die auf dem Knacken des STI-Jackpots basieren. Vielmehr wird es eine solide, notwendige Problemlösung entlang der normaleren DUI-Innovationsrouten sein, die den größten Teil des sozialen Wertes ausmacht, der sich aus dem Ökosystem der Enerprise ergibt.

Zweitens war eine der Erkenntnisse der evolutionären Komplexitätstheorie (ECT), die eine nützliche Rolle in der Unternehmensökosystemtheorie gespielt hat, die von Kauffman (2008) in Bezug auf die Evolutionsrate der Neuheit (Innovation oder kreatives Artefakt oder Dienstleistung) angebotene, dass sich das wirtschaftliche Gefüge der Ökosysteme, in die sie eingebettet sind, selbst entwickelt und komplexer wird in Bezug auf ihre Machtgesetze von Umfang und Umfang. je größer ist die Chance für weitere, sich beschleunigende Neuheiten, auch die der Unternehmen. Dies knent an unseren Verweis auf eine einst utopische Idee an, die als Nebenprodukt eine enorme Freisetzung sozialer Energie in das Unternehmertum verursachen könnte, nämlich die Idee eines "Grundeinkommens". Auf nationaler und regionaler Regierungsebene wird nun ausreichend darüber nachgedacht, dass es zum ersten Mal seit den Levellers und Diggers zaghaft in der Praxis angewendet wird. Die enorme Belastung der öffentlichen Ausgaben, die durch 
das enorme Gewicht der Gesundheits- und Sozialdienste und die Armeen von Beamten, die $40 \%+$ des nationalen BIP verwalten, ausgelöst wird, ist so groß, dass die Bedingungen angemessen werden, um das "Grundeinkommen" für alle Bürger in Betracht zu ziehen. Denken Sie daran, dass das vorgeschlagene monatliche Schweizer Stipendium £ 1.700 (\$ 2.400) an jeden Bürger gezahlt wird, ein Satz von $£ 20.400$ (\$28.000). Das Schweizer Gesundheits- und Wohlfahrtsbudget (inklusive Verwaltung) Die erwachsene Bürgerbevölkerung beträgt 4,8 Millionen. Das Schweizer BIP betrug 2016475 Milliarden US-Dollar (KKP); Das BIP für Gesundheit und Wohlfahrt beträgt $36 \%$. So werden etwa 171 Milliarden US-Dollar des BIP für Gesundheit und Wohlfahrt ausgegeben. Im Vergleich dazu werden 140 Milliarden US-Dollar an vorgeschlagenem Grundeinkommen vorgeschlagen, eine grobe Ersparnis pro Jahr von 31 Milliarden US-Dollar für die Schweizer Steuerzahler. Ein Teil davon sind private Ausgaben, aber dennoch sind die öffentlichen Ausgaben mit zwei Dritteln für die soziale Sicherheit beträchtlich. Der Punkt hier ist, dass von diesen Gesundheits- und Wohlfahrtsökosystemen, von denen erwartet werden kann, dass sie dynamische, interaktive, effiziente und effektive Unternehmen sind, trotz unvermeidlicher Start-ups und noch reiferer Misserfolge, ein Anstieg der Unternehmensgründungen zu erwarten ist.

Schließlich bietet "Vielfalt", insbesondere "verwandte Vielfalt" (einschließlich "offenbarter verwandter Vielfalt") in Bezug auf "Unternehmensökosysteme", die in Bezug auf die Suche, Auswahl und Strukturierung des Unternehmenspotenzials in Bezug auf Ökosystemmöglichkeiten spezifiziert wurden, eine neue und "pfadschaffendere" Entwicklung neuer und diversifizierter Geschäftsmöglichkeiten als "pfadabhängigere" Denkweisen über Unternehmertum. In Bezug auf die separaten und unverwechselbaren, aber innovationsorientierten Systeme, mit denen zukünftige, strukturierte Unternehmensökosysteme (EE) interagieren können, bietet das gemeinsame Modell von RIS und EE einen leistungsfähigen konzeptionellen und praktischen Rahmen für zielgerichtete Maßnahmen. In "Grand Challenge"-Kontexten wie "Alterung", "Gesundheitsanstiege" und "Ambient Assisted Living", die jeweils enorme Belastungen für Steuergelder und öffentliche Investitionen darstellen, könnte es kein besseres globales Experiment geben, mit dem die Entwicklung von Instrumenten für komplexität sowohl in RIS als auch in EE beginnen könnte. Dementsprechend unterstreicht die interessante Idee der "Enterprise Ecosystems" oder, wenn gewünscht, "entrepreneurial ecosystems" das Potenzial neuer Möglichkeiten, nicht nur die "Tugenden", sondern auch den "Wert der Vielfalt" zu entwickeln, da letztere noch näher an der Schnittstelle zwischen Patient und Anbieter liegt als die RIS-Perspektive.

\section{TRANSLATED VERSION: PORTUGUESE}

Below is a rough translation of the insights presented above. This was done to give a general understanding of the ideas presented in the paper. Please excuse any grammatical mistakes and do not hold the original authors responsible for these mistakes.

\section{VERSÃO TRADUZIDA: PORTUGUÊS}

Aqui está uma tradução aproximada das ideias acima apresentadas. Isto foi feito para dar uma compreensão geral das ideias apresentadas no documento. Por favor, desculpe todos os erros gramaticais e não responsacule os autores originais responsáveis por estes erros.

\section{INTRODUÇÃO}

Esta apresentação de artigo analisa um esforço para contrabalançar o crescente surgimento da hegemonia neoliberal na economia política regional no início do atual milênio. Em um artigo de Cooke (2002) que existia como um guia para os formuladores de políticas da UE para uma abordagem alternativa à "nova teoria do crescimento" e especialmente aquela variante que enfatizava a "teoria do crescimento endógeno" (Romer 1990) foi formada a ideia de "crescimento generativo". Isso deriva de raízes teóricas muito diferentes e igualmente profundas às da perspectiva neoclássica. Basicamente, criticou o individualismo radical, o determinismo e a linearidade da perspectiva neoclássica e preferiu uma 
abordagem evolutiva, socialmente interativa e não linear à economia política. É claro que a história revela que nenhuma atenção foi dada ao meu conselho pela equipe de formulação de políticas da UE. Eles seguiram os vários Goldman Sachs, para não dizer Lehman Brothers, rebanhos como seus mentores políticos dos EUA tinham aprovado. Isso incluiu a inclinação para trás para permitir que os comerciais de tecnologia corporativa de alta tecnologia dos EUA retenham impostos, serviços de saúde e outros contratos públicos a preços de transferência, e benefícios de livre comércio (TTIP) para acumular aos seus concorrentes rivais. O mais chocante foi a forma como as empresas e o governo dos EUA primeiro advertiram e depois intimidaram a UE com ameaças legais contra "vantagem competitiva" em seus próprios quintais (Myant 2015). O acordo comercial proposto alimentaria preocupações de que, por exemplo, os fundos soberanos do Serviço Nacional de Saúde do Reino Unido poderiam ser atacados por empreiteiras privadas usando o sistema judicial ISDS (investor state dispute settlement). Isso simula uma espécie de "cesarismo econômico" que lembra o domínio da apropriação na Grã-Bretanha romana para a era contemporânea da competitividade global por todos os meios possíveis.

Ao reconsiderar o crescimento maciço da ideologia neoclássica e o crescimento ainda maior do mau funcionamento das relações sociais, os efeitos catastróficos da guerra, dos refugiados e da migração que assolam o espaço econômico global de regiões, nações e territórios amplos, a primeira coisa a registrar foi o declínio da ação cooperativa, socialmente interativa e colaborativa nos assuntos econômicos de forma mais geral de 2000 a 2016. Isso pode estar mostrando sinais crescentes de decadência, mas o poder do vicelíder do capital sobre as principais instituições das economias global, do bloco e nacional continuou a se fortalecer. Onde podem ser encontrados sinais do surgimento de predisposições mais evolutivas, socialmente responsivas e de aprendizagem? Entre alguns podem ser contados o crescimento contínuo, ainda que não avassalador, da inovação coletiva e da atividade empreendedora. Ambos dependem significativamente da formação de conhecimento, exploração, exame e exploração entre "estranhos atrativos" que muitas vezes são capazes de interagir através das fronteiras (indústrias, setores, clusters) para criar e inovar com diferentes tipos de atores criativos. Embora possam competir, eles também são capazes de colaborar para criar algo de valor social comercializável. Além disso, embora os dados de patentes de IST de inovação tecnológica sejam cuidadosamente registrados, a contribuição muito maior para o valor inovador da atividade de "fazer, usar a interação" é amplamente reconhecida como sendo sob ou mesmo não registrada da mesma forma. É como "material escuro" no universo, ao nosso redor, mas difícil de ver. Empreendedores e inovadores são os grandes responsáveis por esse "material escuro".

\section{CONCLUSÃO}

Chegamos aos três principais pontos de discussão da análise anterior, como um prelúdio para os três pontos conclusões aos quais estão relacionados. O primeiro deles abre um modo mais holístico, integrado e comum de prática econômica institucional. Isso privilegia - em certa medida - o empreendedor individualista que busca lucros, embora prefira as características mais neutras e diversas do empreendimento como veículo para análise e realização de valor. Isso se correlaciona com a noção de "crescimento generativo", um processo de aprendizagem mais evolutivo, comunitário e socialmente interativo do que a teoria mais estreita do crescimento endógeno. Trata-se de reducionista, bem como individualista e determinado por seu foco principal no motivo do lucro por excelência. No entanto, o "crescimento endógeno" não é maior do que a compra de tecnologia "fora da prateleira", o que é em grande parte capricho do julgamento de risco do empreendedor. Em contrapartida, o "crescimento generativo" explora coletivamente a força em números que um modo mais desinteressado de prática empresarial, ganhando com "bens de clube" e "propagação de risco" como descrito para os distritos industriais clássicos marshallianos. Isso ressoa com a disposição de aprendizagem mais comum e interativa dos sistemas regionais de inovação. Estes são ainda mais desinteressados em suas inovações do que os empreendedores estão em seus lucros. Isso porque eles são impulsionados por uma metodologia de resolução de problemas ou descoberta que pode não, mesmo no longo prazo, depender do contexto, alcançar a rentabilidade. Para sobreviver, eles podem ter que construir novos modelos de negócios que tenham o caráter de estratégias de "seguro de conhecimento" (por exemplo, start-ups de biotecnologia; em escala corporativa, a fixação de 
preços pelo Estado desempenha uma função comparável para a geração de energia nuclear). Assim como os ecossistemas empreendedores/corporativos e os sistemas regionais de inovação podem ter bons e maus exemplares de prática aceitável. Isso associado aos ecossistemas empreendedores está muito mais próximo do mercado de propósito, onde para sistemas de inovação o desempenho sub-ideal está inclinado a agir "com boa vontade" mas ser traído por questões de "informação assimétrica", seleção adversa ou "falha de mercado" que podem acabar com a política fora de curso (Stam 2015).

O segundo ponto de discussão é o quanto o ecossistema empresarial ainda desenvolveu, conceitualmente, muito menos em termos de prática real, capacidades em relação a um dos conceitos-chave deste artigo, expressos em seu título, ou seja, desenvolver uma metodologia de pesquisa para lidar com a diversidade ou, especificamente, no contexto deste artigo "variedade relacionada" (incluindo "variedade relacionada revelada"). Isso implicaria múltiplos projetos de pesquisa, eles mesmos diversos, para traçar mutações corporativas ou biografias empresariais que se revelam não como empresas de lucro indiferenciadas, mas como entidades econômicas purposivas. Estes construiriam suas ações purposivas em relação a outros empreendimentos que facilitaram a realização da maior finalidade, como a lâmpada (LED), o smartphone ou a articulação protética do quadril ou joelho. Há relativamente pouco dessa "análise ancestral" em comparação com os volumes de estudos da "empresa mais antiga do mundo" em estudos de empreendedorismo. Pode haver algum risco ligado à formulação de estudos intersetores ou intersetos "cross-over", mas do ponto de vista da inovação, muita inovação tem esse caráter de "variedade relacionada", incluindo valor "revelado" que ocorre por acidente tanto quanto design. No entanto, é a pureza que justifica o risco de busca e potencial de seleção.

E como ponto de discussão final, no que diz respeito à nossa discussão sobre estática e dinâmica em relação aos ecossistemas empresariais. Parece provável que a perspectiva mais evolutiva do ecossistema possa, se abordada e resolvida metodologicamente, auxiliar o surgimento de um perfil mais dinâmico da gestão empresarial, se não o empreendedorismo em si. O equilíbrio estático e fazer apostas seguras pela inclinação de imitar ou "enxame" em termos schumpeterianos, em vez de se envolver em uma verdadeira prática de tomada de risco, apoiando a inovação purposive para o valor social está atrasando o estudo no campo. Armado com a criatividade implícita no cross-over e em outros tipos de empreendimentos empreendedores/empresariais (EDP) torna-se mais dinâmico porque está embutido em ecossistemas emergentes e em evolução. Estes são estruturados quando viáveis em plataformas de cluster que celebram sua variedade relacionada revelada, por exemplo, envolvendo empresas petrolíferas em conversas com empresas de alimentos sobre energia de biocombustíveis, ou relojoeiros e produtores de motores de combustão encontrando novos mercados e usuários de tecidos de assentos de nanotecnologia para evoluir novos materiais para uniformes médicos antissépticos. A empresa criativa estará viva para tais (imprevisíveis) revelados resultados relacionados à variedade porque celebra a mudança dinâmica.

Em termos de principais conclusões, uma das práticas mais fortes, teoricamente e analíticas é que, se o empreendedorismo é difícil e a gestão empresarial é circunscrita pelo problema da "satisficing" e da "racionalidade delimitada", os ecossistemas corporativos são beneficiados por sua propensão de networking, suas "comunidades de prática" regionais e os bens livres de aprendizagem interativa dos pares do ecossistema, sejam as empresas ot intermediários. Assim, embora seja um campo imaturo, decorrente da "prática de reforço" em vez de uma bem argumentada descoberta de pesquisadores e observação prática do mundo real, ela tem um potencial de valor para se tornar um contribuinte dinâmico da teoria e da política. É importante ressaltar que agora está claro que o empreendedorismo é diferente de natureza e tipo de atividade inovadora. Mas também é claro que muito esforço realizado nos ecossistemas corporativos será menos do que o desempenho da empresa de alto crescimento com base na atingindo o jackpot STI. Em vez disso, será sólido, necessário, solução de problemas ao longo das rotas de inovação mais normais do DUI, que respondem pela maior parte do valor social decorrente do cenário do ecossistema enerprise.

Em segundo lugar, um dos insights da teoria da complexidade evolutiva (ECT) que tem desempenhado um papel útil na teoria do ecossistema empresarial tem sido o oferecido por Kauffman (2008) em relação à taxa de evolução da novidade (inovação ou artefato criativo ou serviço) é que, à medida que o tecido econômico dos ecossistemas dentro dos quais eles estão incorporados, evoluem e se tornam mais complexos em termos de suas leis de poder de escala e escopo, maior é a oportunidade para mais, acelerando a 
novidade, incluindo a das empresas. Isso se conecta à nossa referência ao que antes parecia uma ideia utópica que - como subproduto - poderia causar uma enorme liberação de energia social na empresa, ou seja, a ideia de uma "renda básica". O pensamento suficiente está agora a ser dado em níveis governamentais nacionais e regionais que está começando a ser aplicado provisoriamente na prática pela primeira vez desde os Levellers e Diggers. Tal é a vasta carga dos gastos públicos ocasionados pelo enorme peso dos serviços de saúde e assistência social e pelos exércitos de servidores públicos que administram o que pode equivaler a $40 \%$ + do PIB nacional que as condições estão se tornando adequadas para considerar "renda básica" para todos os cidadãos. Tenha em mente que o salário mensal suíço proposto é de £ 1.700 (US \$ 2.400) pagos a cada cidadão, uma taxa de £ 20.400 (US \$ 28.000). A população suíça de saúde e bem-estar (incluindo administração) é de 4,8 milhões. O PIB suíço em 2016 foi de US\$ 475 bilhões (PPP); O PIB de Saúde e Bem-Estar é de $36 \%$. Assim, aproximadamente US\$ 171 bilhões do PIB são gastos em Saúde e Bem-Estar. Isso se compara com US\$ 140 bilhões em receita básica proposta, uma economia aproximada por ano de US\$ 31 bilhões para os contribuintes suíços. Parte disso é despesa privada, mas mesmo assim, com dois terços dedicados à seguridade social, o gasto público é substancial. A questão aqui é que uma onda de formação empresarial poderia ser esperada a partir desses ecossistemas de Saúde e BemEstar, que podem ser empreendimentos dinâmicos, interativos, eficientes e eficazes, apesar de inevitáveis falhas inevitáveis e até mesmo mais maduras.

Finalmente, a "variedade" especialmente "variedade relacionada" (incluindo "variedade relacionada revelada") em relação aos "ecossistemas corporativos" tendo sido especificada em termos de busca, seleção e estruturação do potencial empresarial em relação às oportunidades do ecossistema, oferece uma nova e mais "criação de caminhos" de novas e diversificadas oportunidades de negócios do que formas mais "dependentes do caminho" de pensar sobre empreendedorismo. Em relação aos sistemas separados e distintos, mas orientados à inovação, com os quais os ecossistemas corporativos estruturados futuros (EE) podem vir a interagir, o modelo conjunto RIS e EE oferece uma poderosa estrutura conceitual e prática para a ação purposiva. Em contextos de "grande desafio" como "envelhecimento", "surtos de saúde" e "vida assistida ambiente" cada um dos quais colocam enormes cargas sobre os recursos do contribuinte e o investimento público, não poderia haver melhor experiência em escala global sobre a qual começar a evoluir ferramentas para a complexidade tanto no RIS quanto no EE. Assim, com este último ainda mais próximo da interface paciente-provedor do que a perspectiva RIS, a interessante ideia de "ecossistemas corporativos" ou, se preferiram "ecossistemas empreendedores" sublinha ainda mais o potencial de novas oportunidades para o desenvolvimento não apenas das "virtudes" mas do "valor da variedade". 